\title{
Incidence of respiratory viruses in a pediatric population: molecular and epidemiological aspects
}

\author{
Anna Di Taranto', Rosella De Nittis', Mariantonietta Di Stefano',2, Giuseppina Faleo', \\ Maria Rosaria Lipsi', Valeria Delli Carri', Mariangela Pugliese', Teresa Colapietra ${ }^{3}$, \\ Rosaria Agriesti', Raffaele Antonetti' \\ I Dipartimento Patologia Clinica, UOC II Laboratorio Analisi Azienda Mista Ospedaliera Universitaria OO.RR Foggia \\ 2 Dipartimento di Scienze Mediche e Del Lavoro Università degli Studi di Foggia, Italia \\ 3 UOC Pediatria, Azienda Mista Ospedaliera Universitaria OO.RR Foggia
}

Key words: Respiratory viruses, Human Influenza A, HINI

Incidenza di virus respiratori in una popolazione pediatrica: aspetti molecolari ed epidemiologici

\section{SUMMARY}

Introduction: Respiratory infections are not well defined and the etiology is often unknown.

Material and method: four hundred fortynine subjectrs were enrolled in the study; in all patientes there was a suspect of inflammatory diseases of the respiratory tract. At admission, a nasopharyngeal swab was made.

A multiplex PCR was performed after extraction and reverse transcription of viral RNA. The amplified fragments were revealed by using an electrophoresis separation.

Results: Two hundred and four patients (45.4\%) were hospitalized for infection of the upper respiratory tract, I4I (3I.4\%) for lower respiratory infection and the remaining (23\%) for other symptoms.

One hundred fiftyseven (35\%) patients were positive for human influenza A (HINI subtype) and 184 for other respiratory viruses, of which 59 (32\%) gave a positive for respiratory syncytial virus, $42(23 \%)$ for rhinovirus, 31 (17\%) for parainfluenza virus, 12 (6.5\%) for coronavirus, 28 (15\%) for adenovirus and 6 (3\%) for influenza B (3\%) and $6(3 \%)$ for metapneumovirus.

The MI gene sequence of influenza A HINI strains from 12 patients had a high identity with that of the reference virus.

Conclusion: Furthermore HINI and RSV were the main causative agents of acute respiratory infection.

A molecular approach provides an accurate and rapid aetiological diagnosis of viral respiratory infections.

The molecolar features in the MI gene suggested that the HINI influenza strains circulating in Apulia region had a conserved genetic make up.

\section{INTRODUZIONE}

Le patologie dell'apparato respiratorio hanno elevata incidenza e sono una delle più frequenti cause di malattia, invalidità e morte in Italia ed in Europa (9). In particolare, le infezioni respiratorie da virus influenzali determinano il $20-30 \%$ delle morti nei bambini di età inferiore a 6 anni nei paesi in via di sviluppo, mentre risultano responsabili di un significativo aumento della spesa sanitaria nei paesi sviluppati dove le percentuali di mortalità in genere non hanno raggiunto valori di rilievo $(1,4,8,11,12)$.

Tuttavia, dati recenti dell'Health Protection Agency hanno segnalato diversi decessi associati a virus influenza A di tipo H1N1 nel Regno Unito $(2,5,6)$ in soggetti appartenenti a categorie a rischio non vaccinate. Anche in Italia nel 20092010 sono stati registrati incrementi di malattie gravi e decessi associati a tale virus $(3,7,10)$. Ciò ha determinato un maggiore interesse nei confron- ti dei virus respiratori ed in particolare di quelli influenzali da parte dei media e dei sanitari.

La diagnosi eziologica delle infezioni respiratorie risulta a tutt'oggi complessa, in quanto i quadri clinici possono risultare variabili e aspecifici; l'ausilio del laboratorio, pertanto, diviene fondamentale.

Lo scopo di questo studio è stato quello di impiegare saggi di biologia molecolare per la rivelazione dei principali agenti virali di affezioni respiratorie acute in pazienti pediatrici. Altri obiettivi dello studio sono stati quelli di definire la prevalenza dei diversi virus rivelati, verificare gli andamenti stagionali, correlare gli agenti virali con il sesso e le caratteristiche cliniche dei soggetti infettati e indagare sull'aspetto genetico dei virus influenza A H1N1 identificati.

\section{MATERIALI E METODI}

Lo studio è stato condotto nel periodo ottobre

\section{Corresponding author: Anna Di Taranto}

Dipartimento Patologia Clinica, UOC II Laboratorio Analisi Azienda Mista Ospedaliera Universitaria OO.RR. Foggia

V.le Pinto - 7II20 Foggia - Tel.: 088I 732269; Fax: 088I 732060

E-mail: aditaranto@ospedaliriunitifoggia.it 
2009-aprile 2010 su pazienti pediatrici, affetti da flogosi delle vie respiratorie, afferenti al Reparto di Pediatria dell'Azienda mista UniversitariaOspedaliera OORR Foggia.

Il materiale biologico, raccolto mediante tampone naso-faringeo (Copan Flock Technologies srl, Brescia, Italia) e conservato ad una temperatura di $-20^{\circ} \mathrm{C}$ fino al momento dell'esame, è stato sottoposto a indagini virologico-molecolari mediante una multiplex PCR del commercio (Seeplex RV12 ACE Detection, Seegene, Seoul, Korea), in grado di amplificare simultaneamente più segmenti di DNA appartenenti ai diversi virus respiratori (Tabella 1). Per i virus a RNA, il genoma virale è stato retrotrascritto mediante l'utilizzo di un kit del commercio (Revertaid First Strand cDNA Sinthesis Kit, Fermentas).

Il campione è stato stemperato in $600 \mu \mathrm{l}$ di soluzione fisiologica e sottoposto ad una estrazione mediante un kit del commercio (QIAamp MinElute Virus Spin Kit, Qiagen Bruxelles, Belgium).

L'amplificazione è stata condotta su termociclatore (SEEAmp, Seegene, Seoul, Korea) secondo il seguente profilo termico: denaturazione $15 \mathrm{minu}-$ ti a $94^{\circ} \mathrm{C}$, seguita da 40 cicli a $94^{\circ} \mathrm{C}$ per $30 \mathrm{sec}$, $72^{\circ} \mathrm{C}$ per $90 \mathrm{sec}$ ed un'estensione finale a $72^{\circ} \mathrm{C}$ per 10 minuti. Per ogni reazione è stato utilizzato un controllo positivo ed uno negativo.

I campioni risultati positivi per il virus Influenza A sono stati sottoposti alla determinazione del genotipo H1N1 mediante una multiplex PCR del commercio che amplifica un frammento di $262 \mathrm{bp}$ (Seeplex ACE Detection, Seegene, Seoul, Korea). I frammenti amplificati sono stati visualizzati mediante "MultiNa, Microchip Electrophoresis System" for DNA/RNA Shimadzu Corporation, Kyoto, Japan.

I campioni amplificati e tipizzati per il virus H1N1 sono stati sottoposti a purificazione mediante l'utilizzo di colonnine; dopo centrifugazione, il DNA è stato eluito in $20 \mu 1$ di tampone ed ulteriormente centrifugato per 5 minuti a temperatura ambiente a $8000 \mathrm{rpm}$.

Il DNA è stato sequenziato, utizzando il sequenziatore automatico ABI PRISM 310 (Applied Biosystems).

Le sequenze ottenute sono state confrontate con quelle depositate in GeneBANK utilizzando il programma informatico Basic Local Alignement Search Tool (BLAST) della National Center for Biotechnology Information (NCBI).

\section{RISULTATI}

Lo studio è stato condotto su 449 pazienti pediatrici, 254 maschi (56\%) e 195 femmine (44\%) di età compresa tra 93 giorni e 14 anni. Di questi bambini, al ricovero 204 (45.4\%) presentavano sintomi e segni clinici a carico delle alte vie respiratorie, 141 (31.4\%) broncopolmoniti o bronchiti e 99 (22\%) iperpiressia, convulsioni febbrili e/o sintomi a carico dell'apparato digerente.

Di 5 bambini (1\%) non era conosciuta la diagnosi all'ingresso (Figura I).

Dei 449 bambini sottoposti alla ricerca dei virus respiratori 157 (35\%) sono risultati positivi per il virus dell'influenza A sottotipo H1N1 (63 femmine e 94 maschi), 184 (41\%) positivi per altri virus respiratori (98 maschi e 86 femmine) e 108 (24\%) sono risultati negativi alla ricerca dei virus respiratori. Nei 184 bambini positivi per virus respiratori diversi da virus influenza è stato rivelato in 59 $(32 \%)$ casi virus respiratorio sinciziale, in 42 $(23 \%)$ casi per rhinovirus, in $31(17 \%)$ casi virus parainfluenza, in $12(6.5 \%)$ casi coronavirus, in $28(15 \%)$ casi adenovirus, in $6(3 \%)$ casi virus influenza $\mathrm{B}$ e in $6(3 \%)$ casi metapneumovirus (Figura II).

La correlazione tra sintomatologia clinica e agenti rivelati è mostrata in Figura III.

Nel gruppo dei bambini risultati positivi al tampone per virus influenza A H1N1, il 41\% (64 su 157) presentava una sintomatologia a carico delle alte vie respiratorie, il 34\% (53 su 157) a carico delle basse vie respiratorie, 35 bambini (22\%) segni e sintomi clinici non ascrivibili a patologie respiratorie.

Nel gruppo dei bambini risultati positivi ad altri virus respiratori, il 52\% (95 di 184) manifestava una sintomatologia a carico delle alte vie respiratorie, il 33\% (61 su 184 a carico delle basse vie respiratorie e infine il 15\% (28 su 184) presentavano altri sintomi clinici.

I bambini, per i quali non è stato possibile stabilire una diagnosi eziologica, nel 25\% (27 su 108) hanno manifestato sintomi associati alle alte vie respiratorie, nel 42\% (45 di 108) alle basse vie respiratorie ed infine nel 33\% (36 su 108) sintomi e segni clinici a carico di apparati diversi da quello respiratorio.

L'analisi temporale delle diverse infezioni virali ha dimostrato un tipico andamento stagionale delle infezioni respiratorie: il virus Influenza A $\mathrm{H} 1 \mathrm{~N} 1$ è risultato prevalente nei mesi invernali con un picco nei mesi di novembre e dicembre, seguito dai virus respiratorio sinciziale e adenovirus. Rhinovirus è stato rivelato nei mesi di gennaio e febbraio e virus parainfluenza ha mostrato il suo picco in primavera (Figura IV).

Il sequenziamento dei prodotti di amplificazione del gene M1 di 12 ceppi di virus influenza A H1N1 rivelati ha dimostrato un'identità nucleotidica del $97 \%$ con quella del ceppo H1N1 CTF150/2009 di riferimento. Le mutazioni osse- 
vate in posizione 5, 91 e 112 delle sequenze analizzate, tuttavia, non cambiavano la relativa sequenza aminoacidica.

\section{CONCLUSIONI}

I risultati di questo studio indicano che nell'area di Foggia il virus influenza A H1N1 e il virus respiratorio sinciziale sono i principali agenti di natura virale responsabili di infezione respiratoria acuta, con un picco di incidenza tra novembre e febbraio, mentre il virus parainfluenza causa infezioni soprattutto nei mesi primaverili (marzo-aprile) ed i rhinovirus prevalgono nei mesi di gennaio e febbraio.

Dai risultati conseguiti si evince, inoltre, che l'indagine molecolare è fondamentale sia sotto l'aspetto clinico che epidemiologico. Essa, infatti, consente da un lato, di porre una diagnosi eziolo- gia precisa in tempi brevi e, dall'altro, nel caso di virus influenza, attraverso l'analisi del frammento nucleotidico amplificato, di condurre una sorveglianza epidemiologico-molecolare dei ceppi rivelati al fine di identificare precocemente l'insorgenza di fenomeni di mutazione in grado di creare emergenze sanitarie e di conseguenza favorire tempestive strategie preventive e/o terapeutiche.

In questo studio il ritrovamento di 3 mutazioni nel gene M1 di tutti i ceppi di virus influenza H1N1 rivelati da bambini con affezioni respiratorie, incapaci di alterare la sequenza aminoacidica della relativa proteina, suggerisce che il virus circolante nell'area di Foggia possiede un genoma altamente conservato rispetto al ceppo di riferimento.

Tabella I. Virus respiratori, gene bersaglio dell'amplificazione e lunghezze dei relativi frammenti nucleotidici amplificati mediante saggio multiplex-PCR (Seeplex RVI 2 ACE Detection, Seegene, Seoul, Korea).

\begin{tabular}{|c|c|c|c|c|c|}
\hline SET A* & $\begin{array}{c}\text { gene } \\
\text { bersaglio }\end{array}$ & pb & SET B* & $\begin{array}{c}\text { gene } \\
\text { bersaglio }\end{array}$ & $\mathbf{p b}$ \\
\hline metapneumovirus & $\mathrm{F}$ & 749 & virus influenza $B$ & PBI & 754 \\
\hline adenovirus & Pol & 534 & coronavirus OC43 & gpl & 578 \\
\hline corovavirus NL63 & $S$ & 375 & rhinovirus $A / B$ & 5'UTR & 394 \\
\hline virus parainfluenza 2 & $\mathrm{HN}$ & 264 & virus respiratorio sinciziale $\mathrm{A}$ & $\mathrm{F}$ & 273 \\
\hline virus parainfluenza 3 & $\mathrm{~F}$ & 188 & virus influenza $A$ & MI & 206 \\
\hline virus parainfluenza I & $\mathrm{HN}$ & 139 & virus respiratorio sinciziale $B$ & $\mathrm{~F}$ & 143 \\
\hline
\end{tabular}

* L'amplificazione prevede l'utilizzo di due diversi set A e B che includono coppie di primers che amplificano i diversi geni dei virus respiratori, in modo da evitare una cross - reazione tra le coppie di primers.

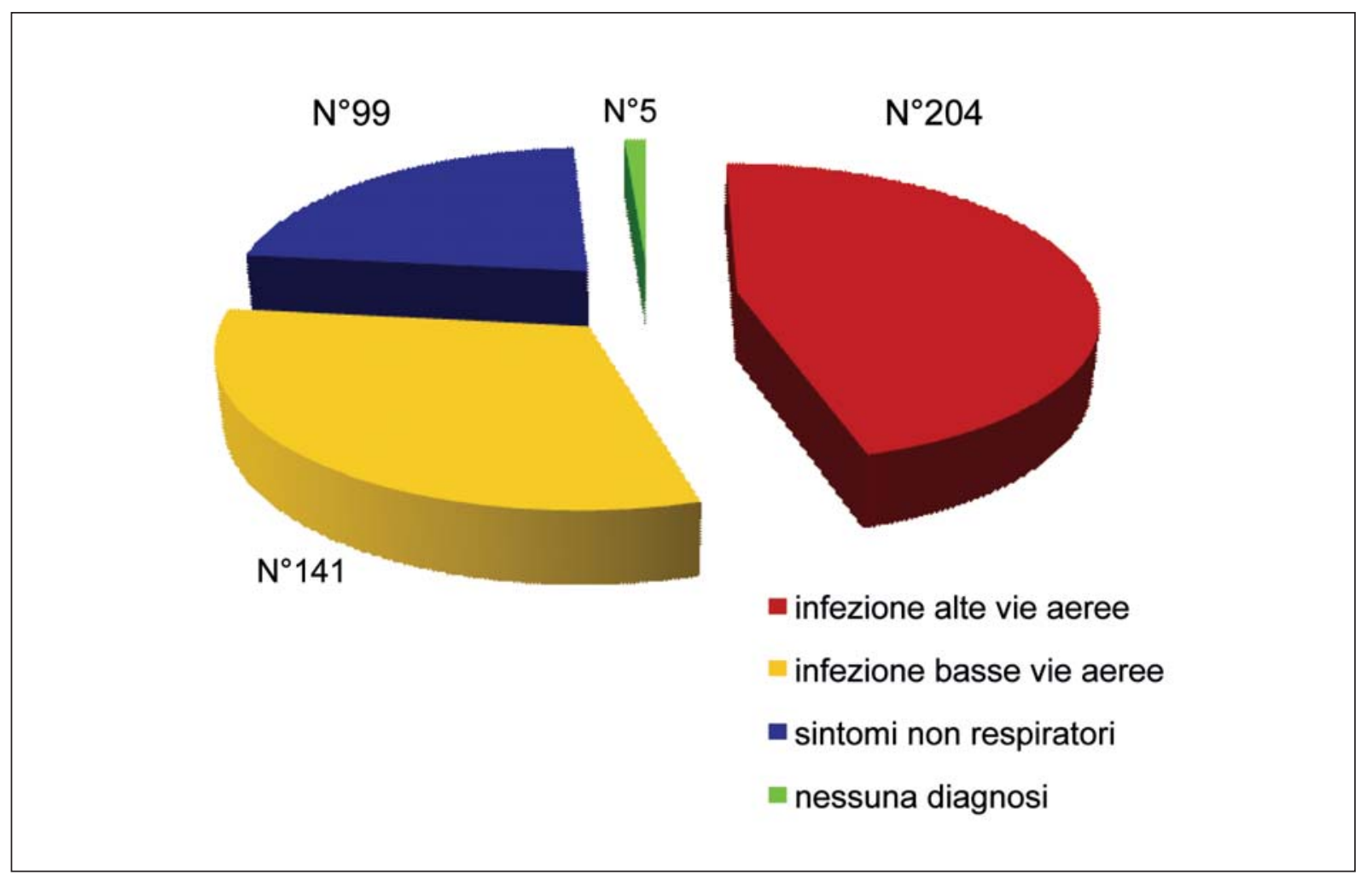

Figura I. Sospetto diagnostico elo situazione clinica al ricovero di 449 bambini (ottobre 2009-aprile 20 I0). 


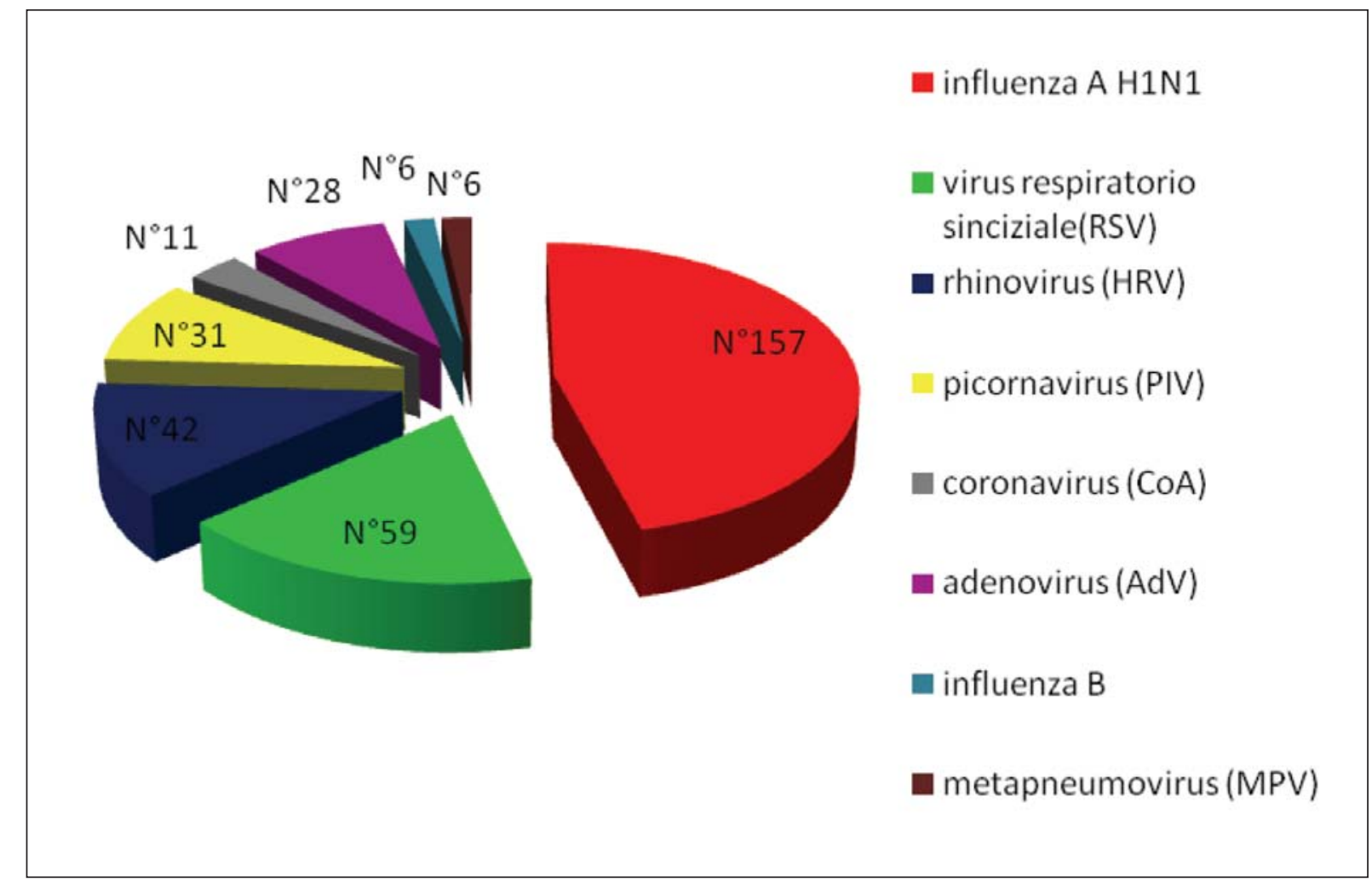

Figura II. Distribuzione degli agenti virali identificati in 340 bambini ricoverati con affezioni respiratorie (ottobre 2009aprile 2010).

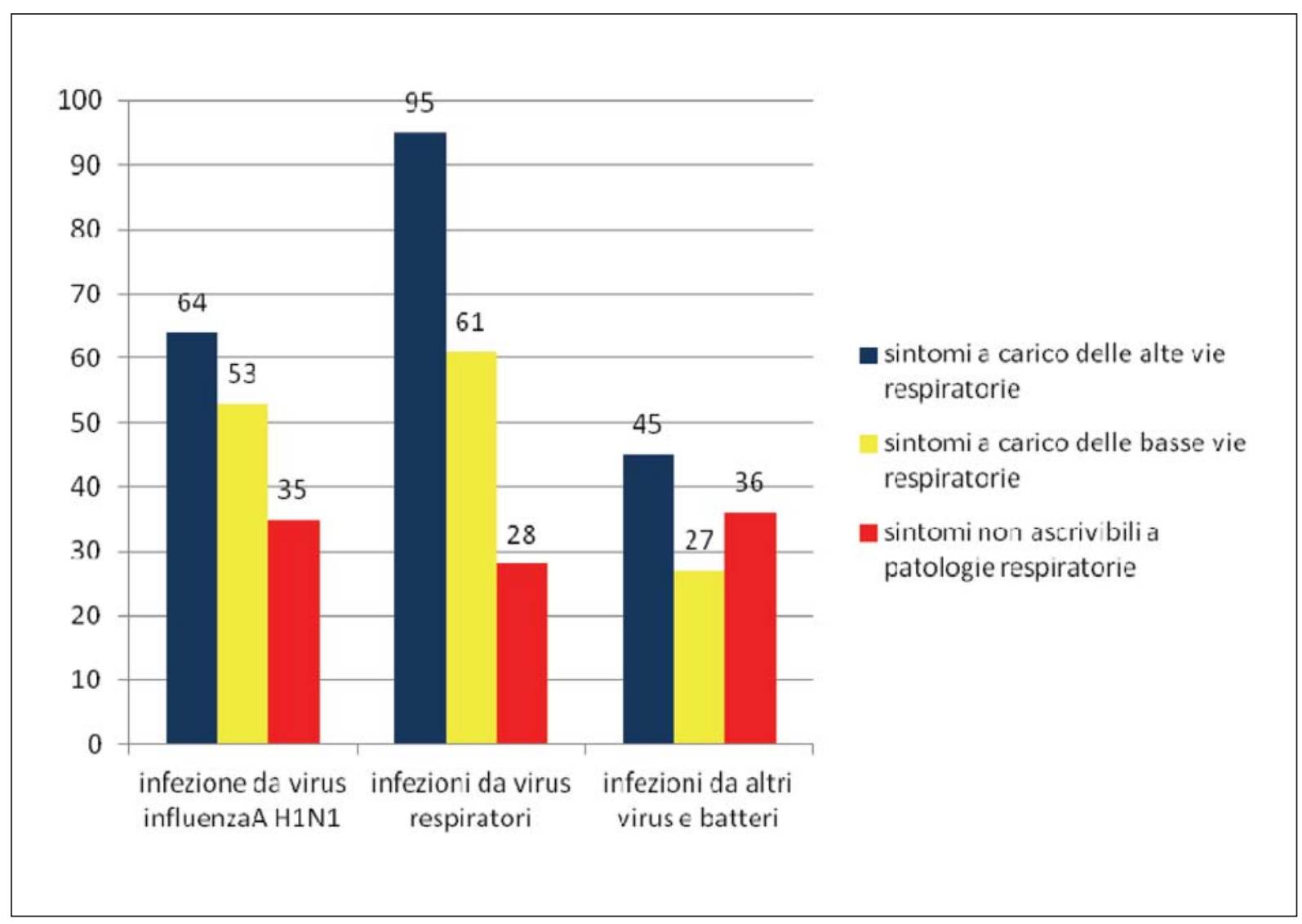

Figura III. Correlazione tra agenti rivelati e sintomatologia clinica. 


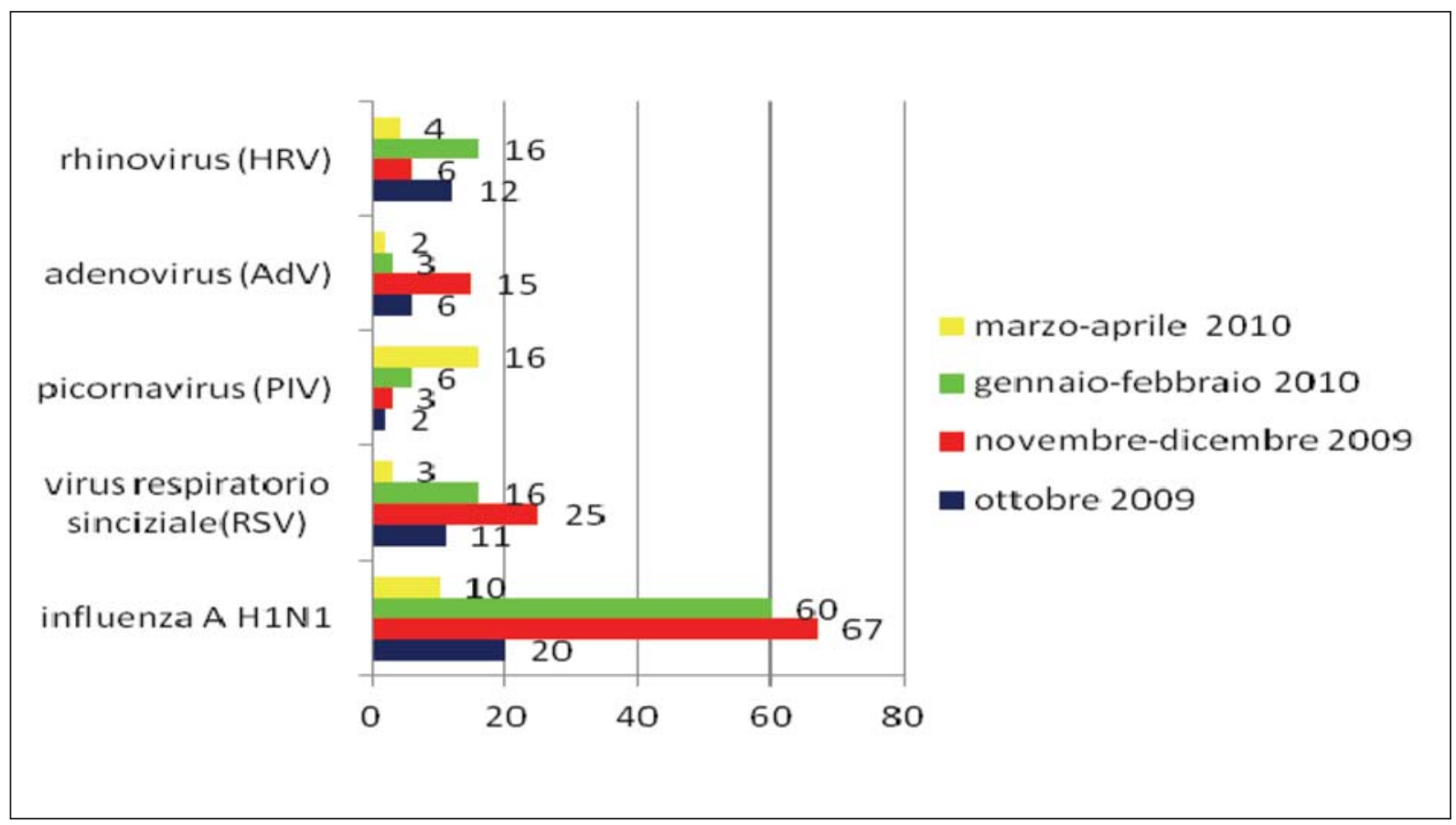

Figura IV. Distribuzione per bimestre dei virus respiratori identificati in 340 bambini con affezioni respiratorie nel periodo ottobre 2009-aprile 2010.

\section{7}

\section{H1N1CTF150/2009}

CEPPO $n^{\circ} 1$ CEPPO $n^{\circ} 2$ CEPPO $n^{\circ} 3$ CEPPO $n^{\circ} 4$ CEPPO $n^{\circ} 5$ CEPPO $n^{\circ} 6$ CEPPO $n^{\circ} 7$ CEPPO $n^{\circ} 8$ CEPPO $n^{\circ} 9$ CEPPO $n^{\circ} 10$ CEPPO $n^{\circ} 11$ CEPPO $n^{\circ} 12$

\section{TGGGAATGGGGACCCGAACAACATGGATAGAGCAGTTAAACTATACAAGAAGCTCAAAAG}

TGGGGATGGGGACCCGAACAACATGGATAGAGCAGTTAAACTATACAAGAAGCTCAAAAG TGGGGATGGGGACCCGAACAACATGGATAGAGCAGTTAAACTATACAAGAAGCTCAAAAG TGGGGATGGGGACCCGAACAACATGGATAGAGCAGTTAAACTATACAAGAAGCTCAAAAG TGGGGATGGGGACCCGAACAACATGGATAGAGCAGTTAAACTATACAAGAAGCTCAAAAG TGGGGATGGGGACCCGAACAACATGGATAGAGCAGTTAAACTATACAAGAAGCTCAAAAG TGGGGATGGGGACCCGAACAACATGGATAGAGCAGTTAAACTATACAAGAAGCTCAAAAG TGGGGATGGGGACCCGAACAACATGGATAGAGCAGTTAAACTATACAAGAAGCTCAAAAG TGGGGATGGGGACCCGAACAACATGGATAGAGCAGTTAAACTATACAAGAAGCTCAAAAG TGGGGATGGGGACCCGAACAACATGGATAGAGCAGTTAAACTATACAAGAAGCTCAAAAG TGGGGATGGGGACCCGAACAACATGGATAGAGCAGTTAAACTATACAAGAAGCTCAAAAG TGGGGATGGGGACCCGAACAACATGGATAGAGCAGTTAAACTATACAAGAAGCTCAAAAG TGGGGATGGGGACCCGAACAACATGGATAGAGCAGTTAAACTATACAAGAAGCTCAAAAG

\section{H1N1CTF150/2009}

$$
\begin{aligned}
& \text { CEPPO } n^{\circ} 1 \\
& \text { CEPPO } n^{\circ} 2 \\
& \text { CEPPO } n^{\circ} 3 \\
& \text { CEPPO } n^{\circ} 4 \\
& \text { CEPPO } n^{\circ} 5 \\
& \text { CEPPO } n^{\circ} 6 \\
& \text { CEPPO } n^{\circ} 7 \\
& \text { CEPPO } n^{\circ} 8 \\
& \text { CEPPO } n^{\circ} 9 \\
& \text { CEPPO } n^{\circ} 10 \\
& \text { CEPPO } n^{\circ} 11 \\
& \text { CEPPO } n^{\circ} 12
\end{aligned}
$$

Figura V. Allineamento di una sequenza nucleotidica di I 8 pb del gene MI di I 2 ceppi di virus influenza A HIN I rivelati in bambini con sindrome respiratoria con la sequenza del ceppo di riferimento HINICTFI50/2009 (ottobre 2009-aprile 2010). 


\section{BIBLIOGRAFIA}

1. Denny FW, Clyde WA Jr. Acute lower respiratory tract infections in non hospitalized children. Journal of Pediatrics 1984; 108 (5): 635-46.

2. European Center for Disease Prevention and Control (2009). Weekly electronic Bulletin Week 32: 03 August 2009-09 August 2009. Available: http://ecdc.europa.eu/en/activities/surveillance/EISN/New letter/SUN_EISN_INFL_Bulletin_2009week32.pdf. Accessed20̄10 Märch 8 .

3. European Center for Disease Prevention and Control (ECDC) Announced cumulative number of confirmed fatal cases of 2009 pandemic Influenza A (H1N1) in EU and EFTA updated 3 May 2010 Stockholm, Sweden.

4. Glezen WP, Frank AL, Taber LH, et al. Parainfluenza virus type 3 : seasonality and risk of infection and reinfection in young children. Journal of Infectious Desease 1984; 150 (6): 851-7.

5. Health Protection Agency (2009) Pandemic (H1N1) 2009 in England: an overview of initial epidemiological findings an implications for the second wave 2009 December 2.

6. Health Protection Agency (2010) Epidemiological report of pandemic (H1N1) 2009 in UK. Accessed 2010 November 1.
7. Influenza Umana da nuovo virus A/H1N1-aggiornamento 12 e13. 2009 May 4 (Human Influenza due to the novel A/H1N1 virus - Updates 12 and 13,4 may 2009) Rome: Ministry of Labour, Health and social Policies. Communication DGPREV.V/19971/P/I.4.c.a.9 from 4 May 2009 Italian.

8. Kumar D, Humar A. Respiratory viral infections in transplant and oncology patients. Infectious Disease Clinics of North America 2010; 24 (2): 395-412.

9. Kumar D, Michaels MG,Morris MI, et al. Outcomes from pandemic influenza A H1N1 infection in recipients of solid-organ transplants: a mulicenter cohort study. The Lancet Infectiuos Diseases 2010; 10 (8): 521-6.

10. Rizzo C, Rosa M, Bella A, et al. Response to the 2009 influenza A (H1N1) Pandemic in Italy. Euro Surveillance 2010; 15 (49): pii=19744.

11. Vilchez RA, Dauber J, McCurry K, et al. Parainfluenza virus infection in adult lung transplant recipients: an emergent clinical syndrome with implications on allograft function. American Journal of Transplantation 3. 2003; (2): 116-20.

12. Vilchez RA, Dauber J, McCurry K, et al. The epidemiology of parainfluenza virus infection in lung transplant recipients. Clinical Infectious Disease 2001; 33 (12): 2004-2008. 